\title{
Signals for invisible matter from solar - terrestrial observations
}

\author{
Sergio Bertolucci ${ }^{1,2, a}$, Konstantin Zioutas ${ }^{1,3, b}$, Sebastian Hofmann ${ }^{4}$, and Marios Maroudas ${ }^{3}$ \\ ${ }^{1}$ CERN, Geneva, Switzerland, \\ ${ }^{2}$ INFN, LNF, Italy, \\ ${ }^{3}$ University of Patras, Patras, Greece, \\ ${ }^{4}$ Munich, Germany
}

\begin{abstract}
Gravitational lensing of invisible streaming matter towards the Sun or the Earth could be the explanation of puzzling solar/terrestrial phenomena. We have analyzed solar flares, EUV emission and also the global ionization content of the Earth atmosphere. Assuming that this invisible matter has some form of interaction with normal matter and that there exist preferred directions in its flow, then one would expect an enhanced activity at certain planetary longitudes, which is also observed. The broad velocity spectrum of the assumed constituents makes it difficult at this stage to identify the origin of the stream(s) or the nature of its constituents.
\end{abstract}

\section{Introduction}

The detection of the constituents of dark matter (DM) is one of the central challenges in modern physics. While large scale DM distribution in space is relatively uniform, also local streams have been discussed [1]. The existence of such streams of invisible constituents could be at the origin of the puzzling active Sun or the annual anomaly of the ionization of the Earth atmosphere. We assume here that their triggering is due to planetary lensing of invisible matter stream(s). This scenario is totally different from the models based on tidal forces, which have been attempted with very little success since the discovery of the first large flare some 155 years ago. If this seminal idea holds, there will be ways to explore it further in the future, due to its implications in other ongoing DM searches. A detailed description of the reasoning behind this idea is given in ref. [2]. Here we present also new results.

\section{The Observations}

\subsection{Solar Flares}

In this work we provide a statistical analysis of time (=position in the ecliptic) distributions of the inner 3 planets and the Moon, in association with a) the daily occurrence of $6091 \mathrm{M}$-class and the 491 X-class flares (1976-2015) [3], b) the continuous full disk solar emission in the EUV (1999-2015),

\footnotetext{
ae-mail: Sergio.Bertolucci@cern.ch

be-mail: Konstantin.Zioutas@cern.ch
} 
and c) the electron content of the Earth ionosphere (1995-2012) [4]. Interestingly, for comparison, this period includes the extreme solar minimum of 2008-2009.

The heliocentric longitudinal distribution for Earth, Venus and Mercury of M- and X-class Flares is shown in Figure 1. For the Earth, the peak around $45^{\circ}\left(33^{\circ}\right)$ shows the strongest clustering of such solar energetic bursts, while the peaks at $255^{\circ}$ and in particular that at $291^{\circ}$ are also of statistically significant. In all the planetary spectra no correction was performed for their orbital eccentricity, which is $6 \%, 2 \%$ and $223 \%$ for Earth, Venus and Mercury, respectively. Venus and Mercury show a strong and wide peak around $260^{\circ}-270^{\circ}$ in longitude as well as a second one at $\sim 150^{\circ}$. Interestingly, a strong excess around $250^{\circ}-290^{\circ}$ is happening in all 3 planetary longitudinal distributions, despite the fact that Mercury, Venus and Earth have gone through the same longitudes mostly at different times. In the case of Mercury, a smaller peak appears around $33^{\circ}$. Due to the large orbital eccentricity of Mercury, the expected modulation has been subtracted. The derived residual spectrum makes the aforementioned peaks better visible (Figure 1).

As next, we introduced in the analysis code planetary spatial correlations. A possible combined lensing efficiency might enhance the observed effects on the Sun or the ionosphere. In fact, this has been also observed with statistically significant differences both in intensity and in spectral shape (see Figures 2 and 4). Remarkably, the excess observed with M-Flares and EUV (Figures 2) peaks at exactly at the same values around $241^{\circ}$ and $290^{\circ}$. A similar behavior is also shown by the X-Flares, despite the poorer statistics (see [2]).

Figure 3 shows additional results obtained with the flaring Sun and its full disk EUV emission as well as a correlation between both. Surprisingly, the EUV irradiance preceding the onset of 7 huge Xclass Flares is suppressed for a period lasting 1-2 months. It is intriguing that this suppression depends on the planetary configuration (not shown), which might be a relevant signature for the trigger of the unpredictable flares.

\subsection{The lonosphere}

The total electron content (TEC) of the ionosphere depends primarily on the solar EUV irradiance, but its variations show anomalies, which have not been understood since several decades (see [2]). For example, the measured TEC during December exceeds that around June, which cannot be explained by the annual solar irradiance modulation due to the varying Sun-Earth distance. We investigated the possibility that these anomalies could be connected to planetary lensing, using the same reasoning described above.

Figures 4 shows the results obtained for combined planetary constraints of the global electron content of the ionosphere, derived from an uninterrupted sequence of 6573 daily averaged TEC measurements (1995-2013) [4]. Also this data taken period includes the extremely deep solar minimum between 2008 and 2009, which induced a more quiet behaviour of the electron content of the ionosphere. As before, the time binning of the TEC data is equal to 1 day. Surprisingly, we observed correlations with the position in the ecliptic of Mercury and Venus, including also the orbital positions of the Moon around the Earth. The first two columns in Figure 4 show the longitudinal distribution of Mercury and Venus, requiring a second planet (among the inner 3 planets) to be in a wide heliocentric longitudinal range $20^{\circ}-140^{\circ}$ vs. $200^{\circ}-320^{\circ}$. Three out of four plots refer to the solar maximum period (1997-2006), while a similar comparison is shown for the extreme solar minimum period. Since the derived rates are normalized to 1 day, longitudinal modulations due to the planetary eccentricity are corrected. Considering long observation time periods of 10 or even 18 years, the derived distributions should be rather isotropic. This is, however, in contrast with observation.

We then addressed the annual TEC anomaly at the solstices, selecting the TEC data in $30^{\circ}$ segments around December and June. Figure 4 (right, UP) shows the TEC distributions of these data 
as a function of the Moon phase. Note that during the Earth's propagation by $30^{\circ}$ in longitude, the Moon has completed one geocentric orbit. Taking into account the 223 orbits the Moon has performed around the Earth during the 18 years described by the dataset, randomly occurring TEC excursions should average out, or at least both distributions in Figure 4 (right, UP) should have similar shape. Figure 4 (right, DOWN) shows the calculated difference between the two distributions, exhibiting a modulation amplitude by a factor of 6 . Remarkably, the position of the maximum coincides with the New Moon. This correlation fits well the scenario of this work, assuming a stream of invisible matter, with speed of about $50 \mathrm{~km} / \mathrm{s}$, coming from the direction of the Sun gravitationally focused by the interposed Moon onto the Earth.

\section{Discussion}

By analyzing the occurrence of X- and M-flares during the last 4 solar cycles and the full disk EUV irradiance of the Sun we find a strong evidence that the occurrence of these phenomena is strongly modulated by the position of Earth, Venus and Mercury around their heliocentric longitude. A preferred direction around $270^{\circ}$ is common to all three planets, when their lensing effect is studied independently. The effect is further enhanced, as we show for the case of Mercury and Venus, when one takes into account the relative position of the two planets. This observation supports our working hypothesis that the activity of the Sun is triggered by influx of invisible matter and that this matter has some preferred direction or stream, which gets gravitationally lensed by the planets.

In addition, averaged daily GPS TEC measurements of the Earth ionosphere show also a marked planetary correlation, supporting our lensing scenario, which is further reinforced by the observation of the effect of the position of the Moon in modulating the TEC of the ionosphere. We bring to the attention that the anomalously high electron content of the ionosphere in December coincides with the alignment Galactic Center - Sun - Earth, i.e. the same direction around $270^{\circ}$ previously described and that this effect becomes even more enhanced when the Moon is aligned around the same direction and interposed between the Sun and the Earth.

The identification of the nature of the predicted slow moving stream is left for future work: of course, one has to suppose that this invisible matter has some form of interaction with normal matter, but at this point we cannot infer any useful indication of its nature. Also, the (re)-analysis of the experiments searching for DM following our approach might give unexpected results. 

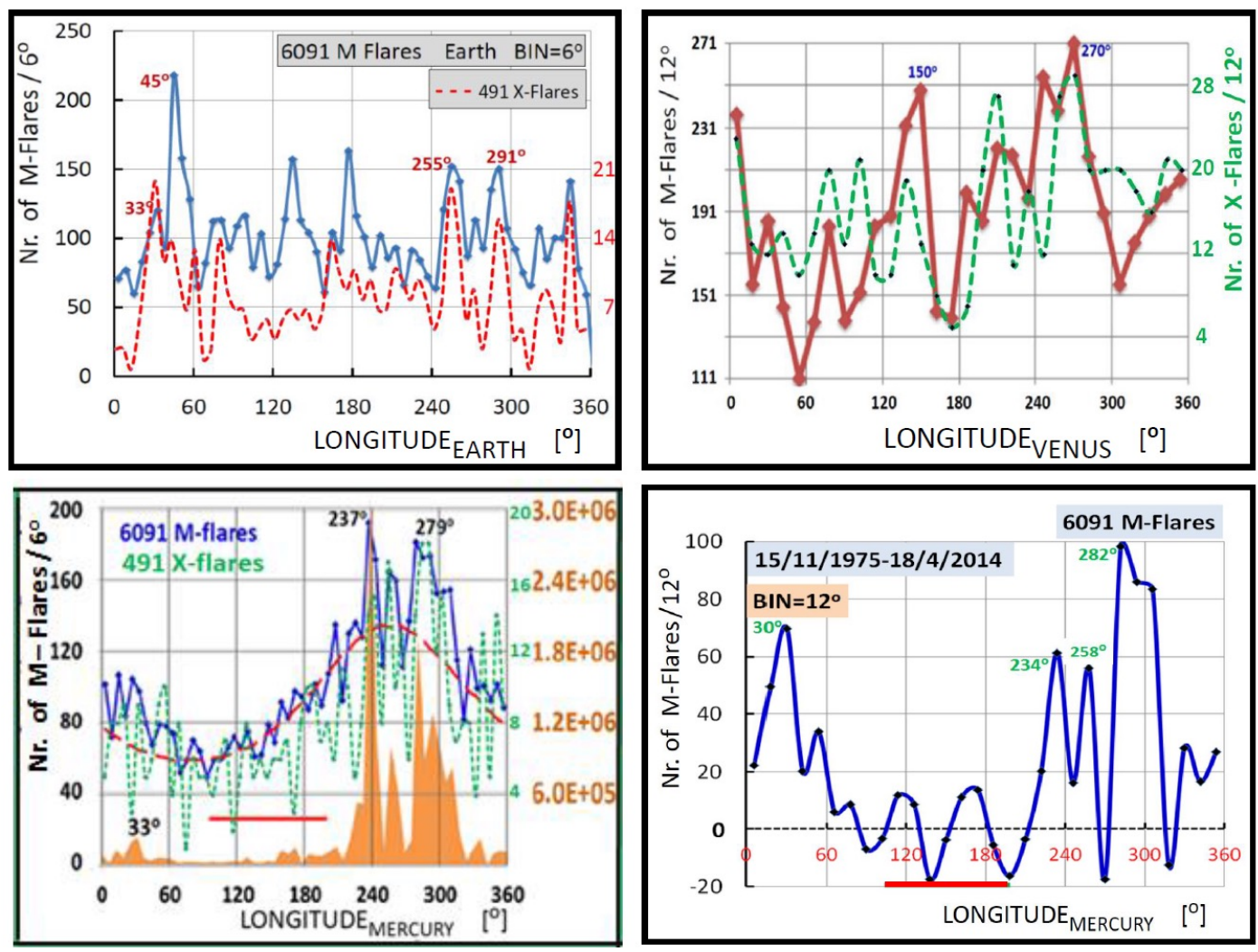

Figure 1. UP, Earth (left), Venus (right): Number of M-class Flares and X-class flares (dashed lines) as a function of the heliocentric longitude of the Earth (01/01/1976 - 05/05/2015) and the Venus (15/11/1975 18/04/2014). DOWN, left: Number of M-class flares (blue) and X-class flares (dashed) as a function of Mercury heliocentric longitude of the $6091 \mathrm{M}$-class Flares. The dashed red line is the normalised stay time due to Mercury orbital eccentricity. The horizontal red bar shows the region used for normalization. In orange is shown the "multiplication spectrum" of the 4 solar cycles (M-Flares). On the right is shown the residual spectrum (blue red dashed on the left), with the 3 peaks around $30^{\circ}, 240^{\circ}$ and $290^{\circ}$ becoming better visible. Note, that we use $B I N=6^{\circ}$ (left column) and BIN $=12^{\circ}$ (right column). 


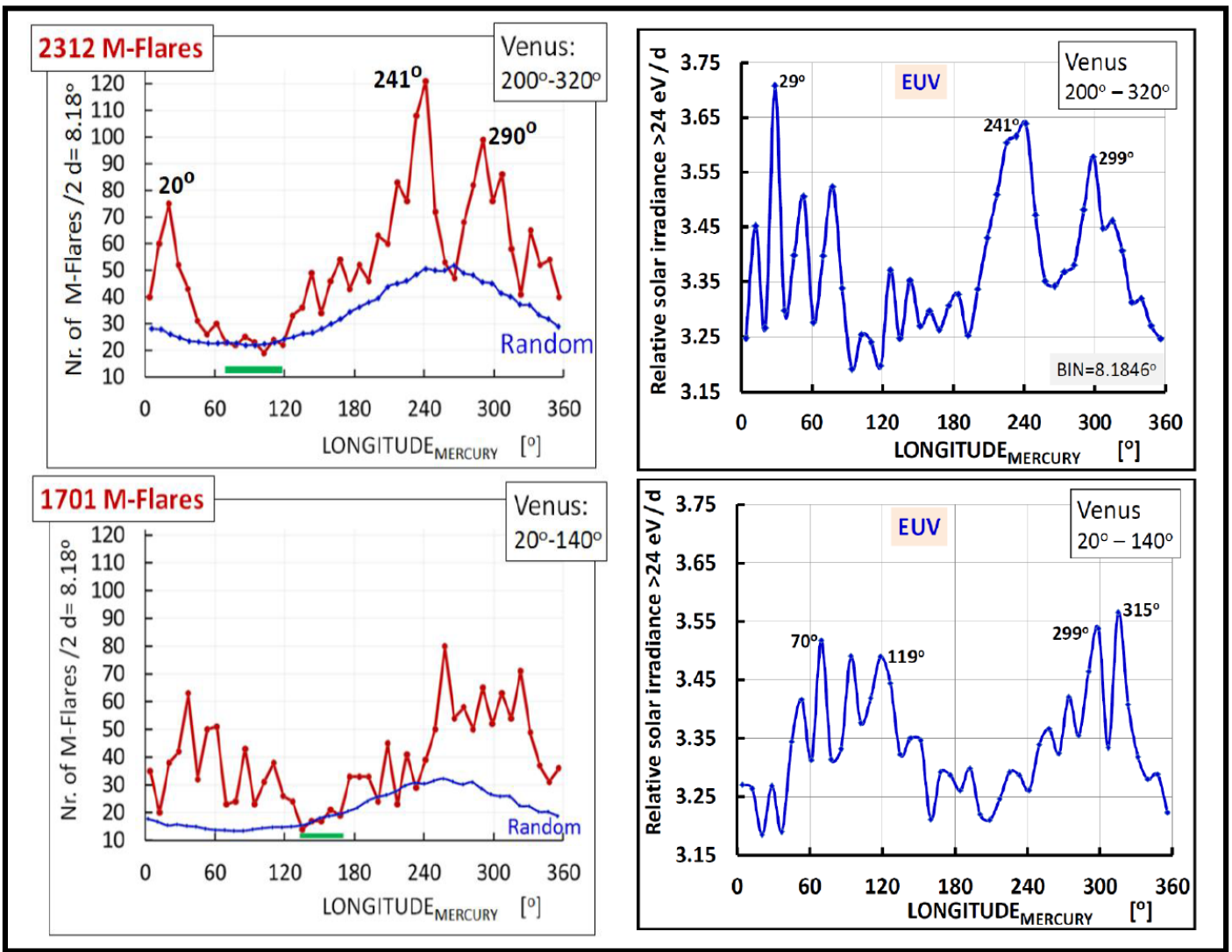

Figure 2. UP: Distributions of M-class Flares (left) and full disk EUV irradiance (right) as a function of Mercury heliocentric longitude with the constraint of Venus being at longitude between $200^{\circ}-320^{\circ}$. DOWN: The same plots for Venus being between $20^{\circ}$ and $140^{\circ}$. The smooth blue lines represent the expected normalized number of flares if equally distributed in time due to the large eccentricity of Mercury. The green bar shows the region used for normalisation. The EUV spectra are time normalised and therefore the eccentricity effect is out. 

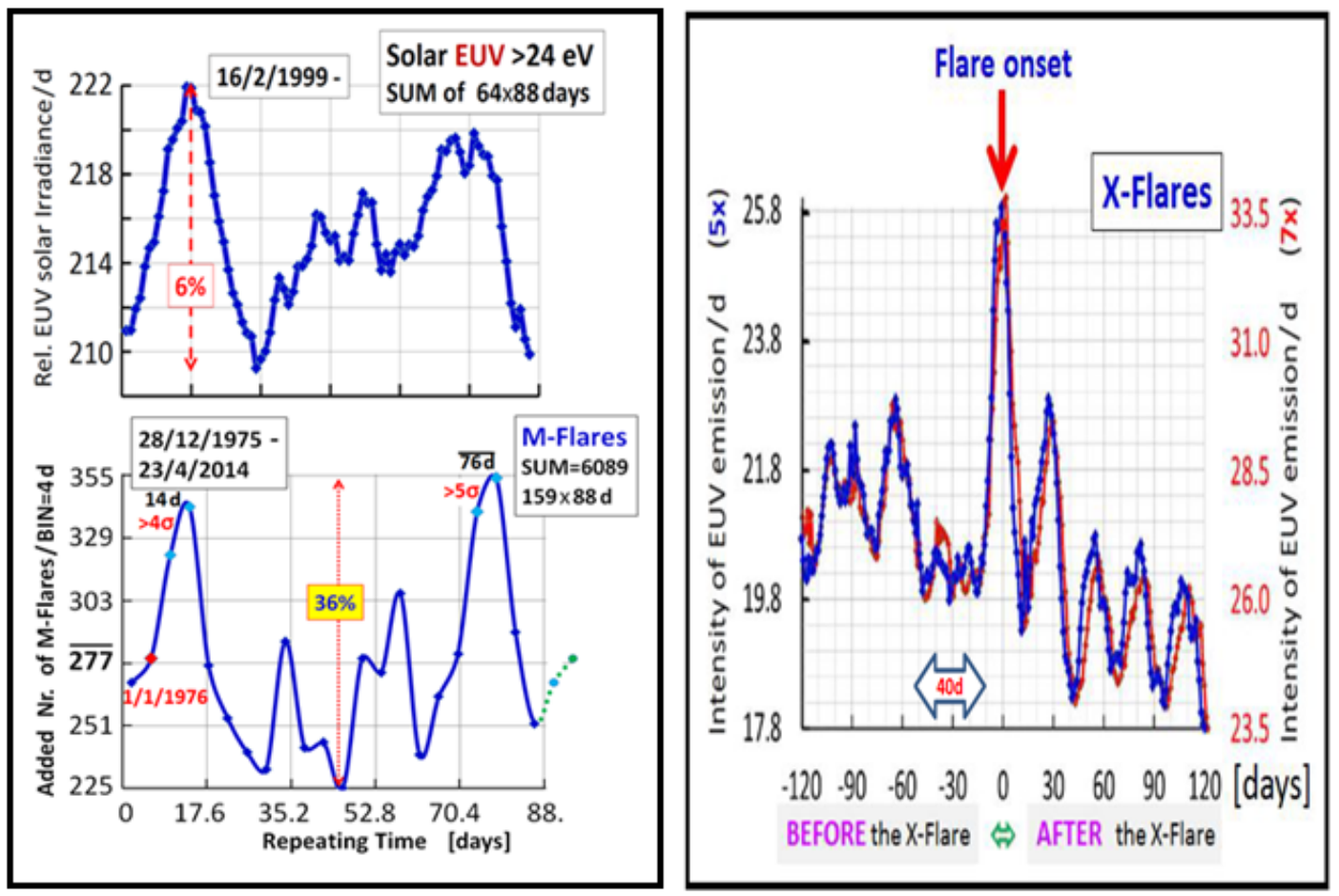

Figure 3. LEFT, UP: The sum of the daily averaged full disk solar EUV emission in 64 consecutive time intervals of 88 days (1999-2015). LEFT DOWN: The sum of M-class Flares averaged over 4 days in 159 consecutive intervals of 88 days (1976-2014). It is apparent the similar spectral shape of both solar activities. RIGHT: The start time of the biggest 7 X-class Flares during the previous solar cycle (1996-2007) is used as the reference point (time $=0$ ) for the emission of full disk solar EUV during 4 months before and after the flare onset, The flares occurred far from each other, excluding double counting effects. The gap of about 40 days before the flare "explosion" is intriguing. Note, for $2 \mathrm{X}$-flare pairs, being too close to each other, an averaged onset time was used; the initial correlation remains unaffected (blue line) 


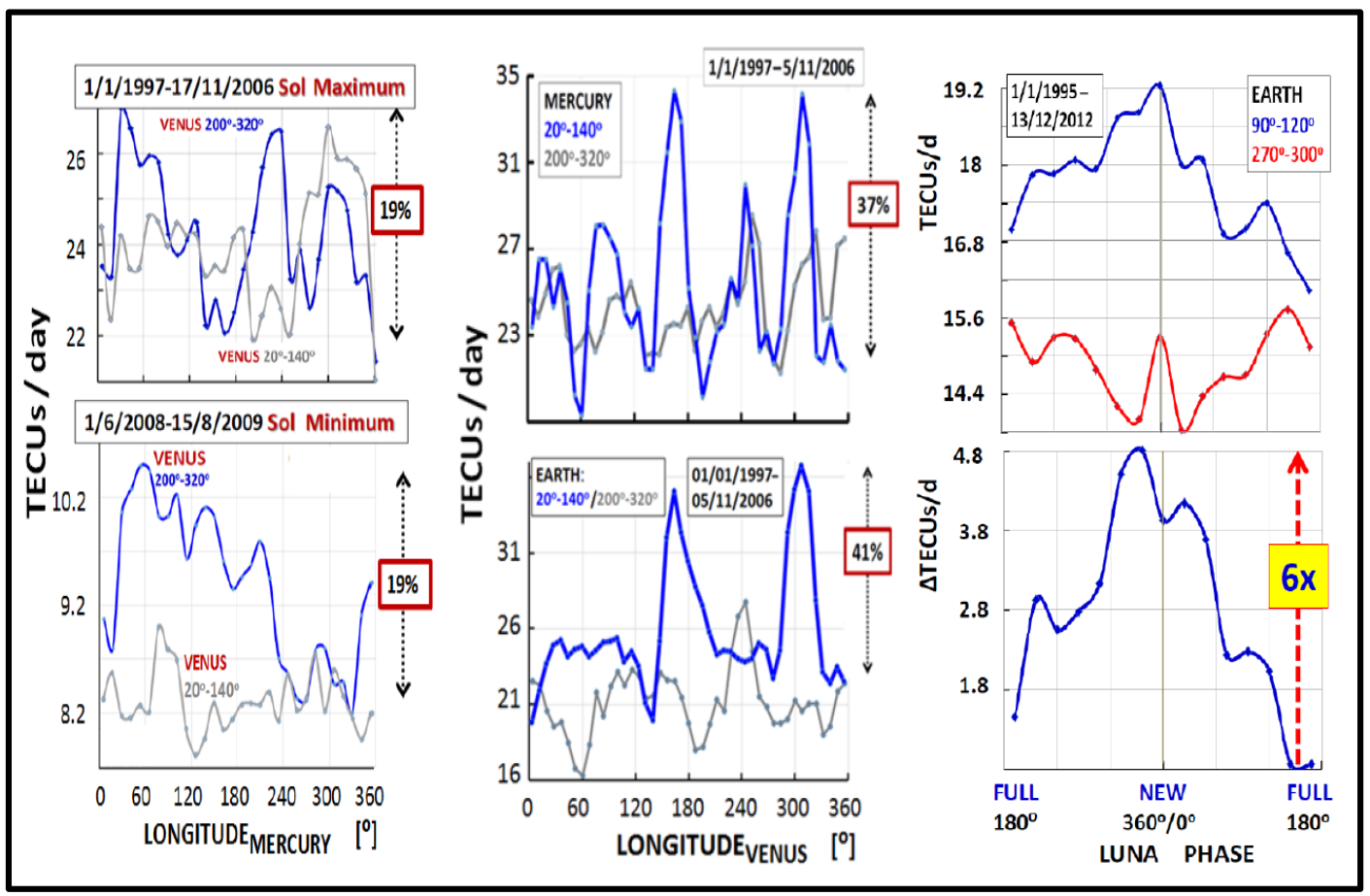

Figure 4. The daily measured longitudinal distributions of Earth's atmospheric total electron content [1] for different planetary configurations and time periods of the solar cycle. LEFT: The total electron content [TECUs] as a function of Mercury heliocentric longitude during the solar maximum period 1997-2006 (UP) and during the recent extremely deep solar minimum 2008-2009 (DOWN). The thick blue and the thin grey lines are associated with Venus being in one of the two opposite $120^{\circ}$ wide orbital arcs. MIDDLE: TECUs as a function of the Venus longitude during the same solar maximum requiring Mercury (UP) and Earth (DOWN) to be in one of the $120^{\circ}$ segments. RIGHT: TECUs as a function of the Moon Phase, while the Earth is in one of the two $30^{\circ}$ orbital segments around the solstices (UP) and the difference between the winter-summer solstices (DOWN). Note, the Moon is between the Earth and the Sun at New Moon. 


\section{References}

[1] C. A. J. O'Hare and A. M. Green, "Directional detection of dark matter streams," Phys. Rev. D 90 (2014) no.12, 123511, doi:10.1103/PhysRevD.90.123511 [arXiv:1410.2749 [astro-ph.CO]], and references therein.

[2] S. Bertolucci, K. Zioutas, S. Hofmann, M. Maroudas, "The Sun and its Planets as detectors for invisible matter," Submitted for publication (2016), [arXiv:1602.03666v4], and references therein.

[3] Astrid Veronig, private communication 2014.

[4] Leonid Didkovsky, private communication 2014. 